\title{
Palmar Fasciitis and Polyarthritis Syndrome: A Rare Paraneoplastic Syndrome in a Patient With Prostate Carcinoma
}

\author{
Anouk G. de Boer ${ }^{\mathrm{a}, \mathrm{d}}$, Ruth Klaasen ${ }^{\mathrm{b}}$, Marlies C. van der Goes ${ }^{\mathrm{c}}$, \\ Haiko J. Bloemendal ${ }^{\mathrm{a}}$
}

\begin{abstract}
A 73-year-old patient was seen in our hospital for treatment of metastatic adenocarcinoma of the prostate $\left(\mathrm{pT}_{1 \mathrm{a}} \mathrm{N}_{0} \mathrm{M}_{1 \mathrm{a}} \mathrm{R}_{0}, B R C A-2\right.$ gene mutation). Prostatectomy and regional radiotherapy were performed and goserelin, a luteinizing hormone-releasing hormone (LHRH) analog, had been started because of disease progression. Castration-resistant progressive disease developed, and enzalutamide was added. A decrease of the prostate-specific antigen (PSA) level was achieved. Before the start of enzalutamide, the patient developed bilateral pain and stiffness of both hands combined with thickening of the hands. The symptoms progressed rapidly to bilateral flexion and extension contractures. The patient became unable to tie his shoelaces and had to use adjusted cutlery to eat. Consultation of the rheumatologist, X-rays, ultrasound and palmar skin biopsy of the hands were performed. The clinical picture resembles descriptions of "palmar fasciitis and polyarthritis syndrome" (PFPAS), a rare paraneoplastic syndrome. Positive effects of immunosuppressive medication have been reported in some cases. In our patient, treatment with oral prednisone ( $30 \mathrm{mg}$ daily) showed no effect, therefore treatment was switched to methylprednisone pulses and methotrexate. PFPAS is an uncommon paraneoplastic syndrome characterized by rapid onset of bilateral arthritis of the hands, fasciitis of the palms, progressive stiffness and contractures. The scarcity of knowledge about PFPAS makes it difficult to recognize it at an early stage. As a paraneoplastic syndrome, it has been linked to various malignancies. Thus far, PFPAS has been described in only two other cases of prostate cancer.
\end{abstract}

Manuscript submitted June 5, 2020, accepted June 23, 2020

Published online August 6, 2020

aDepartment of Internal Medicine, Meander Medisch Centrum, Amersfoort, Netherlands

bDepartment of Rheumatology, Meander Medisch Centrum, Amersfoort, Netherlands

'Department of Rheumatology and Clinical Immunology, University Medical Center Utrecht, Utrecht, Netherlands

${ }^{\mathrm{d}}$ Corresponding Author: Anouk G. de Boer, Department of Internal Medicine, Meander Medisch Centrum, Maatweg 3, 3813 TZ Amersfoort, Netherlands. Email: ag.de.boer@meandermc.nl

doi: https://doi.org/10.14740/jmc3522
Keywords: Palmar fasciitis; Polyarthritis; Paraneoplastic; Prostate cancer

\section{Introduction}

Palmar fasciitis and polyarthritis syndrome (PFPAS) is an uncommon paraneoplastic syndrome. It is characterized by rapid onset of bilateral arthritis of the hands and fasciitis of the palms. PFPAS causes diffuse painful swollen hands and is characteristically recognized by progressive stiffness and contractures [1]. These progressive flexion contractures can lead to a loss of hand function.

PFPAS is mostly described as a paraneoplastic syndrome in patients with ovarian carcinoma [1]. Thus far, PFPAS has been described in only two other cases of prostate cancer $[2$, 3].

In this case report we describe a patient with progressive prostate cancer who developed PFPAS during treatment of his disease. We summarize the clinical characteristics and possible therapies for PFPAS.

\section{Case Report}

A 73-year-old male presented for the treatment of an adenocarcinoma of the prostate with a solitary metastasis in one inguinal lymph gland $\left(\mathrm{pT}_{1 \mathrm{a}} \mathrm{N}_{0} \mathrm{M}_{1 \mathrm{a}} \mathrm{R}_{0}, B R C A-2\right.$ gene mutation). A robot-assisted prostatectomy was performed followed by radiotherapy on the inguinal regions. Despite this therapy, the prostate cancer proved recurrence for which goserelin was started. One year after the prostatectomy progressive disease was noted with new retroperitoneal tumor depositions. The prostate-specific antigen (PSA) level was low at that moment, $3.8 \mu \mathrm{g} / \mathrm{L}$. Enzalutamide (androgen receptor antagonist) was added to the treatment with continuation of goserelin. A decrease of PSA was achieved, $0.36 \mu \mathrm{g} / \mathrm{L}$.

In the same period, just before the start of enzalutamide, the patient developed symptoms of bilateral pain and stiffness of his hands. It started in both hands at the same moment. He also experienced thickening of the dorsal skin of his hands. 


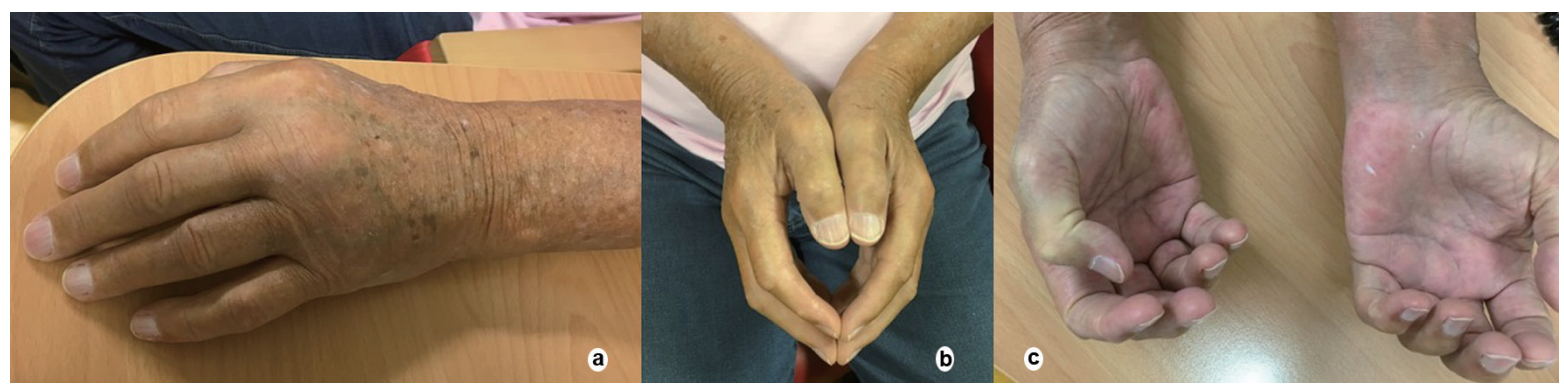

Figure 1. (a) Diffuse thickening of all fingers, mostly around the MCP joints. (b) Full extension of both hands demonstrating "extension contractures". (c) Full flexion of both hands demonstrating "flexion contractures". MCP: metacarpophalangeal.

The symptoms progressed rapidly to bilateral flexion contractures and the impossibility to fully bend or extend his fingers. The patient could not tie his shoelaces and had to use adjusted cutlery to eat.

Physical examination showed diffuse thickening of all fingers, mostly around the metacarpophalangeal (MCP) joints (Fig. 1). Bilateral, nodules were palpable on the palmar side of the hands. Flexion and extension contractures were present in both hands. Scleroderma from fingertips to MCP joints was seen, without other features of systemic sclerosis (no Raynaud's phenomenon, telangiectasia, calcinosis or signs of organ involvement). There were no signs of arthritis in the other joints. X-ray of his hands showed a slight osteoarthritis, but no other signs of ossal pathology. Ultrasound of the hands showed normal tendon sheaths, no sign of Dupuytren's contracture and no hyperemia. Treatment with prednisone (30 mg/day) was started without effect on the symptoms.

The patient was referred for a second opinion to an academic hospital (University Medical Center Utrecht). Physical examination also revealed a positive groove sign in both arms, left more than right (Fig. 2). This is an indentation of the skin above the superficial veins, when the hand is elevated. This sign can be seen with fasciitis. Biopsies of one finger and the palm for pathological research showed fibrosis with a proliferation of fibroblasts in the dermis and subcutis. Intracellular edema was observed in the reticular dermis. In deeper layers of the dermis, an infiltrate with mostly lymphocytes was seen around blood vessels. The findings were not typical for systemic sclerosis but did correspond with the clinical diagnosis of PFPAS.

The diagnosis of PFPAS was confirmed (based on both clinical and pathological findings). Therapy with three methylprednisone pulses of $1,000 \mathrm{mg}$ in combination with methotrexate (MTX) was started. The complaints improved to slightly less swelling and pain of the hands, but the function was still impaired.

Ten months later, therapy with goserelin and enzalutamide was switched to pembrolizumab because of further progression of the disease. Radiotherapy was started because of a new bone metastasis. Meanwhile the MTX was stopped seeing the combination of MTX and radiotherapy can increase the risk of soft tissue necrosis or osteonecrosis. Pembrolizumab was switched to lutetium-prostate-specific membrane antigen (PSMA) radionuclide therapy with a good effect on the PSA- level (drop from 10.7 to $0.68 \mu \mathrm{g} / \mathrm{L}$ ). At the same time, prednisone and MTX were restarted and the complaints of PFPAS

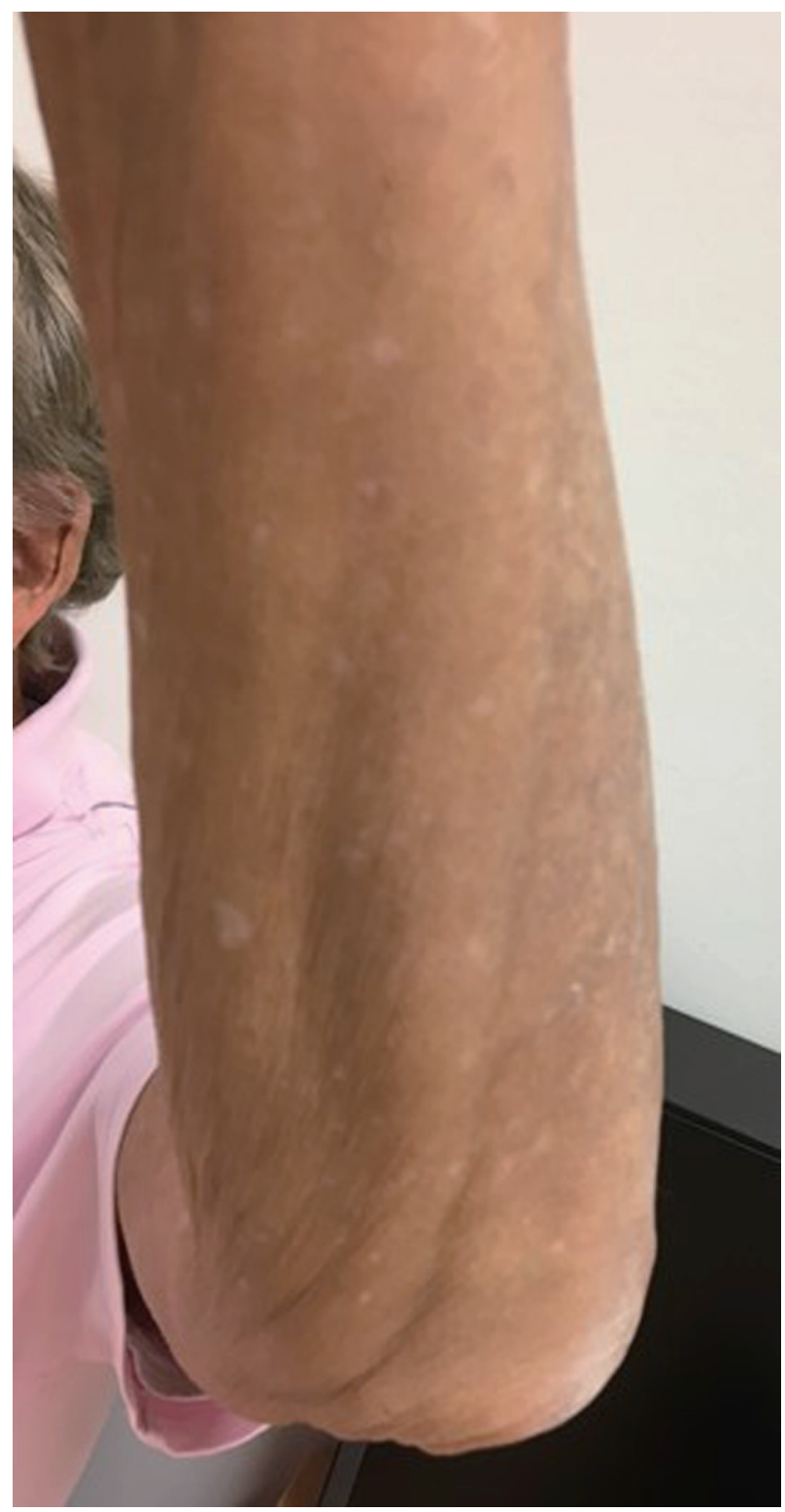

Figure 2. Clinical appearance of "groove sign". 
improved to less swelling of the hands and slightly more functional capacity.

\section{Discussion}

The first paper about PFPAS was published in 1982 by Medsger et al reporting similar symptoms of arthritis and fasciitis in women with ovarian tumors $[1,4]$. PFPAS causes diffuse painful swelling of the hands and is characteristically recognized by progressive stiffness and contractures [1]. These progressive flexion contractures can lead to a loss of hand function. The bilateral swelling of the hands can be accompanied by nodules of the flexor tendons in an advanced stage $[1,5,6]$. These findings and induration of the subcutaneous tissue can be referred to as "woody hands" which describes the hardness and the limited function of the hands. A "groove sign", as was also seen in our case, has been described in other cases with PFPAS. This "groove sign" is more often present in eosinophilic fasciitis, a disease which is also characterized by fibrosis and inflammation of the fascia $[1,7]$. However, in eosinophilic fasciitis the hands are not affected. In PFPAS, arthritis of other joints can also occur, the shoulders being most often involved [8].

Like all paraneoplastic syndromes, PFPAS is a cancer-associated disease without being directly caused by the primary tumor or its metastases. Paraneoplastic syndromes are mediated by a trigger from the immune system against tumor cells or the release of humoral factors such as hormones or cytokines $[4,9]$. The exact pathway of development of PFPAS remains unclear. Typically, in histologic findings fibroblast proliferation is seen which suggests a role of fibroblast activity stimulators, but further research is needed to confirm that hypothesis $[1,4,10]$. Another hypothesis is the influence of hormones in the pathogenesis of PFPAS. Systematic review by Manger et al showed that nearly half of the patients with PFPAS had a malignancy of the reproductive organs. Especially ovarian adenocarcinoma is often seen and described as the most frequent underlying cause of paraneoplastic PFPAS in female patients [1]. This hypothesis could also account for our patient as he had a mutation associated with reproductive organs and hormones: a $B R C A-2$ gene mutation.

Besides ovarian cancer, various malignancies have been described as underlying cause of PFPAS. In a systematic review done in 2014, 100 cases of PFPAS were described and systematically reviewed. Prostate cancer accounted for only two of these cases and after this review no other patients were described with prostate cancer as the underlying cause of PFPAS [1-3]. Our patient therefore counts for the third patient described with PFPAS associated with prostate cancer.

Treatment of PFPAS starts with the treatment of the underlying malignancy, this has shown to have a positive effect on the inflammatory component in some cases. Positive effects of immunosuppressive medication have been reported in some cases. Corticosteroids are often the first choice mainly because of the belief that PFPAS is an immune-mediated disorder. The effect of treatment with corticosteroids is mostly disappointing as the arthritis rarely improves and almost never improvement is seen of the contractures. Sometimes improvement of the swelling and tenderness is seen [2].

In our patient, treatment with oral prednisone $(30 \mathrm{mg}$ daily) showed no effect, therefore treatment was switched to methylprednisone pulses and MTX.

The pulse therapy showed improvement of the swelling but unfortunately, like described in other cases, no improvement of the contractures and the function of the hands was seen. Adequate treatment of the progression of the prostate cancer showed more effect with an improvement of the swelling although the contractures remained invalidating.

Since the contractures caused by PFPAS can be highly invalidating but also unresponsive to therapy, early diagnosis of PFPAS seems to become extra important.

\section{Conclusions}

PFPAS is an uncommon paraneoplastic syndrome characterized by rapid onset of bilateral arthritis of the hands, fasciitis of the palms, progressive stiffness and contractures. The scarcity of knowledge about PFPAS makes it difficult to recognize it at an early stage. As a paraneoplastic syndrome, it has been linked to various malignancies. Thus far, PFPAS has been described in only two other cases of prostate cancer. The most important treatment of PFPAS is the treatment of the underlying disorder, this can create a moderate improvement by a reduction of the inflammation. The fibrosis and contractures appear to be irreversible unfortunately. Treatment with corticosteroids or other anti-immune therapy often leads to disappointing results. Recognition at an early stage remains a challenge.

\section{Acknowledgments}

None to declare.

\section{Financial Disclosure}

Not applicable.

\section{Conflict of Interest}

Not applicable.

\section{Informed Consent}

Patient's informed consent for publication of this report was obtained, written permission not applicable as the patient is not identifiable in the pictures.

\section{Author Contributions}

AGB, RK, MCG and HJB contributed to the design, AGB and 
HJB contributed to the writing and the critical revision of the manuscript. AGB, RK, MCG and HJB gave final approval before submission of the manuscript.

\section{Data Availability}

The data supporting the findings of this study are available from the corresponding author upon reasonable request.

\section{References}

1. Manger B, Schett G. Palmar fasciitis and polyarthritis syndrome-systematic literature review of 100 cases. Semin Arthritis Rheum. 2014;44(1):105-111.

2. Nadal R, McMahan ZH, Antonarakis ES. Paraneoplastic palmar fasciitis and polyarthritis syndrome in a patient with advanced prostate cancer. Clin Genitourin Cancer. 2013;11(4): e15-23.

3. Van den Bergh L, Vanneste SB, Knockaert DC. Palmar fasciitis and arthritis associated with cancer of the prostate. Acta Clin Belg. 1991;46(2):106-110.

4. Medsger TA, Dixon JA, Garwood VF. Palmar fasciitis and polyarthritis associated with ovarian carcinoma. Ann Intern Med. 1982;96(4):424-431.

5. Cox NH, Ramsay B, Dobson C, Comaish JS. Woody hands in a patient with pancreatic carcinoma: a variant of cancer-associated fasciitis - panniculitis syndrome. Br J Dermatol. 1996;135(6):995-998.

6. Alexandroff AB, Hazleman BL, Matthewson M, Black CM, Rytina E, Crawford R, Burrows NP. Woody hands. Lancet. 2003;361(9366):1344.

7. Matteson EL. Groove sign in paraneoplastic palmar fasciitis. J Rheumatol. 1998;25(10):2043-2045.

8. Bremer C. Shoulder-hand syndrome. A case of unusual aetiology. Ann Phys Med. 1967;9(4):168-171.

9. Azar L, Khasnis A. Paraneoplastic rheumatologic syndromes. Curr Opin Rheumatol. 2013;25(1):44-49.

10. Marie I, Cailleux N, Roca F, Benhamou Y, Scotte M, Levesque H. Palmar fasciitis and polyarthritis syndrome. QJM. 2010;103(9):703-704. 